\title{
Large Cell Neuroendocrine Carcinoma of Prostate: A Rare Interesting Case and Literature Review
}

\author{
Reza Mahdavi Zafarghandi, ${ }^{1}$ Mahmood Reza Kalantari, ${ }^{2}$ Alireza Akhavan Rezayat,, ${ }^{3}$ and Amir Abbas
}

Asadpour ${ }^{3}$

${ }^{1}$ Professor of Urology, Mashhad University of Medical Sciences, Mashhad, IR Iran

${ }^{2}$ Associated Professor of Pathology, Mashhad University of Medical Sciences, Mashhad, IR Iran

${ }^{3}$ Assistant professor of Urology, Mashhad University of Medical Sciences, Mashhad, IR Iran

"Corresponding author: Alireza Akhavan Rezayat, Ghaem hospital, Ahmad Abad Ave., Mashhad, IR Iran. Tel: +98-9153148223, Fax: +98-5138012857, E-mail: alirezaakhavan30@yahoo.com

Received 2016 December 29; Accepted 2017 February 12.

\begin{abstract}
Prostate cancer (PC) is one of the most common forms of malignancies and the second cause of cancer death all around the world and the eight causes in Iran. The main portion of PC is adenocarcinoma, in some cases neuroendocrine differentiation occurs. Neuroendocrine prostate cancers ( $\mathrm{NePCs}$ ) incidence varies from $0.5 \%$ to $2 \%$ of all prostate cancers. Large cell neuroendocrine differentiation is very rare. In this study we presented a 71-year-old man with large cell neuroendocrine carcinoma of the prostate.
\end{abstract}

Keywords: Neuroendocrine, Prostate Cancer, Adenocarcinoma

\section{Introduction}

The majority of prostate cancers are adenocarcinoma, but NE differentiation can occur in $10 \%$ of cases in part depending on the number of slides studied and the number of antibodies used (1). Neuroendocrine cells are small subjects in the normal prostate tissue. They are derived from putative stem cells and are androgen receptor negative. In recent studies, it was proposed that they regulate prostate epithelium secretary and proliferative activity. NE cells also can be found in prostate cancers $(2,3)$. The main portion of PC is adenocarcinoma, in some cases neuroendocrine differentiation occurs (4). Neuroendocrine prostate cancers (NePCs) incidence varies from $0.5 \%$ to $2 \%$ of all PCs (3). Neuroendocrine prostate adenocarcinomas are androgen receptor negative (5).

NE tumors of prostate are three groups in regard to world health organization (WHO) histologic classification: 1- focal NE differentiation in conventional prostate cancer (PC); 2- carcinoid tumor; 3- small cell NE carcinoma (1). However prostate adenocarcinomas with NE differentiation are divided into Paneth cell, carcinoid tumor, small cell carcinoma, large cell carcinoma (LCNEC), and mixed form in regard to 2013 prostate cancer foundation classification system (6). Prostate cancer manifestations vary from silent diseases to high grade aggressive forms (3).

Most of neuroendocrine carcinomas in prostate occur in the content of typical adenocarcinoma. Pure neuroendocrine prostate cancers (NePCs) are very rare. The most frequent type of NePCs is small cell carcinoma whereas large cell carcinoma (LCNEC) and carcinoid tumor are extremely rare $(4,5)$. To date, most published LCNECs represented progression from prior typical adenocarcinoma in the content of long standing androgen deprivation therapy (ADT) (6). NE cells and NePCs lack AR and are clinically considered hormone refractory. Overall, NePCs are aggressive and are associated with rapid disorientation and poor prognosis $(1,2)$.

De Novo LCNEC is exceptionally rare particularly in pure forms. Until now, only four series of LCNEC have been published (7). The largest series by Evans et al. described 7 cases with only one pure de novo case. The last case was published by Acosta Gonzalez et al. (2). In this study, we present a 71 year old man with pure de novo LCNEC of prostate.

\section{Case Presentation}

The patient's informed consent has been obtained for the publication of the case reports. A 71-year-old male referred to our clinic with increased dysuria, frequently and urgently for 3 months. He had no systemic signs and symptoms such as fever, night sweats, anorexia, and weight loss. On the digital rectal examination, prostate was enlarged. Prostate specific antigen (PSA) was in the normal range $(0.09 \mathrm{ng} / \mathrm{mL})$. Ultrasonography indicated moderate enlargement of prostate and Benign prostate hyperplasia (BPH). So, tamsulosin was administered for him in re-

Copyright (c) 2017, Nephrology and Urology Research Center. This is an open-access article distributed under the terms of the Creative Commons 
gard to BPH diagnosis. He referred again without any relief after 3 months. His international prostate symptoms score (IPSS) was 22 before treatment and had remained unchanged after three months. He underwent transurethral resection of the prostate (TURP) because of the severity of the symptoms and the patient's desire. Histopathology examination and urine cytology was conducted after TURP. First pathologist reported poorly differentiated carcinoma, so colonoscopy and whole body scan were performed for him and both were negative. Second pathologist proposed LCNEC. The patient refused treatment in this step and presented again after three months with uremia, confusion, and poor general condition. He complained of sever pelvic pain. In the first step, abdomino-pelvic ultrasonography was performed for him and it showed bilateral hydronephrosis. He underwent hemodialysis due to increased creatinine ( $8 \mathrm{mg} / \mathrm{dL}$ ) and ultrasound guided bilateral nephrostomy for obstructive uropathy. A subsequent non-contrast CT confirmed a large pelvic mass with pelvic lymphadenopathy (Figure 1). Tumor was unresectable and he was referred to the oncology department for palliative radiotherapy.

\subsection{Pathological Findings}

The hematoxylin and eosin slides of the TURP specimen showed sheets and a large nest of high grade neoplasm with peripheral palisading, diffusely infiltrating the prostate parenchyma. The neoplastic cells were characterized by large vesicular nuclease with course chromatin and prominent nucleoli and abundant amphophilic cytoplasmic. There were areas of geographic necrosis and high mitotic activity (Figure 2). These features in the current specimen are similar to the histologic description of LCNEC given by Evans et al. (6) in the largest series ever published on LCNEC. Conventional adenocarcinoma was not identified throughout the specimen. Immunohistochemistry (IHC) staining was performed to determine the origin of the neoplasm and the extent of NE differentiation (Table 1). IHC was negative for PSA but was positive for AMACR.

To rule out bladder cancer IHC was performed for P63, CK20, CK 7, and GATA-3. IHC staining with NE markers including chromogranin, synaptophysin, and CD 56 was performed to establish NE differentiation. All of NE markers were strongly and diffusely positive with cytoplasmic staining (Figure 3).

\section{Discussion}

Large cell carcinoma with neuroendocrine differentiation is well known and there are reports of it sporadically found with various origins like lung, cervix, larynx,
Table 1. Molecular Tests Summery

\begin{tabular}{lc}
\hline Marker & Result \\
\hline Synaptophysin & Diffusely positive \\
Chromogranin & Diffusely positive \\
\hline CD56 & Positive \\
AMACR & Diffusely positive \\
\hline CK & Positive \\
\hline CK7 & Negative \\
\hline CK20 & Negative \\
CK5.6 & Negative \\
\hline GATA-3 & Negative \\
\hline PSA & Negative \\
\hline P63 & Negative \\
\hline
\end{tabular}

Abbreviations: AMACR, alpha-methylacyl-coA racemase; $C D$, cluster differentiation; CK, cyto keratin; GATA3, GATAbinding protein3; PSA, prostatic specific antigen

and prostate. These cancers have a distinct pathologic entity and an unfavorable outcome $(8,9)$. LCNEC is a very rare malignancy in prostate. NePCs's typical presentation is symptoms related to prostate enlargement (7). Neuroendocrine markers such as synaptophysin, CD56, and chromogranin are positive in LCNEC. PSA level does not correlate with LCNEC signs and symptoms (8). LCNEC was reported in 7 patients in 2006 from Canada (6). Only one of these patients had de novo LCNEC and he was 69 years old. Tumor was diagnosed incidentally in 5 cases after palliative transurethral prostatectomy (9). PSA level in this patient was lower than $0.1 \mathrm{ng} / \mathrm{mL}$ like our patient. Our patient was not suspected of having NePCs in the first steps and palliative TURP was performed to relieve his symptoms. In a case presented in 2014, a patient was evaluated for increased PSA and was finally diagnosed with LCNEC (2).

Pelvic mass with rapid progression is reported in previous cases $(1,6)$. In our case, huge pelvic mass caused obstructive uropathy and worsened the patient's clinical condition. The mean survival time of NePCs was estimated less than 12 months (3 to 12 months). Our patient was alive in a 6 months follow-up. Death occurs due to metastasis and uropathy, and because of LCNEC delayed diagnosis most cases have metastasis. Our patient, also, had metastasis.

Although androgen-deprivation therapy (ADT) is considered as the main predisposing factor associated with NePCs, some cases have no positive point in their past medical history (10). Okoye introduced a 48 year old man with LCNEC and no history of ADT (7). A total of 6 cases in Evans's study had a history of ADT for prostate adenocarcinoma, but our case had no predisposing factor. LCNEC can 

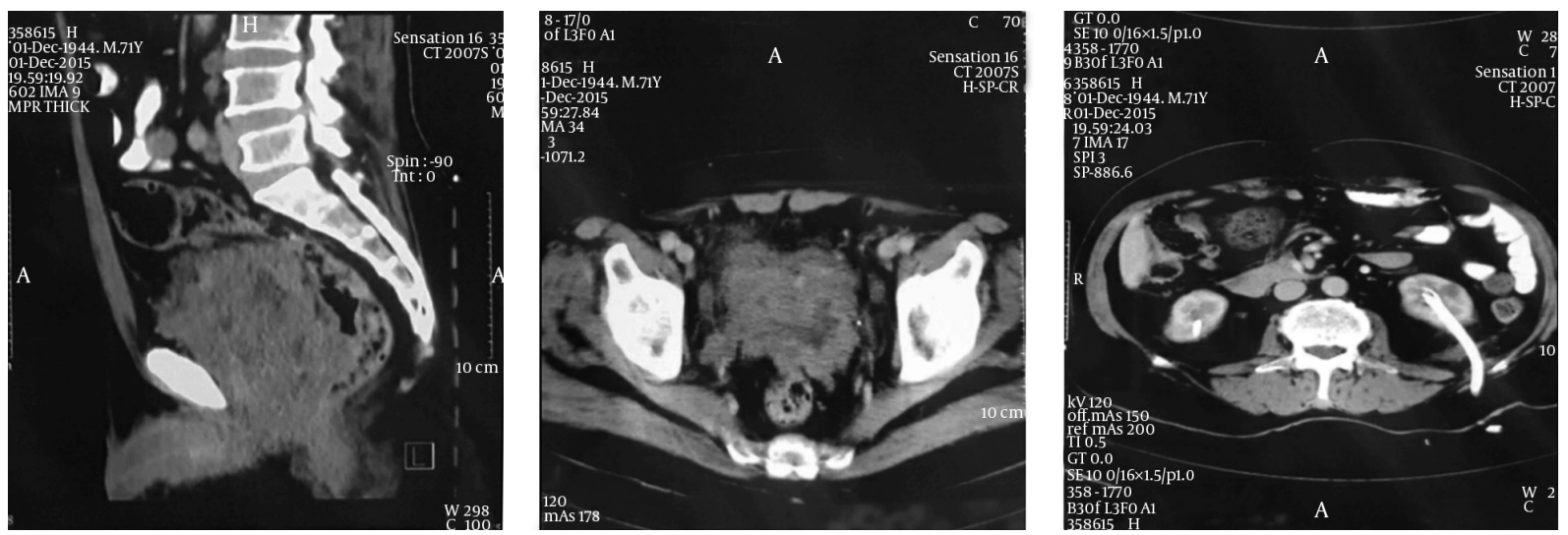

Figure 1. Massive Pelvic Mass with Neural Invasion and Nephrostomy
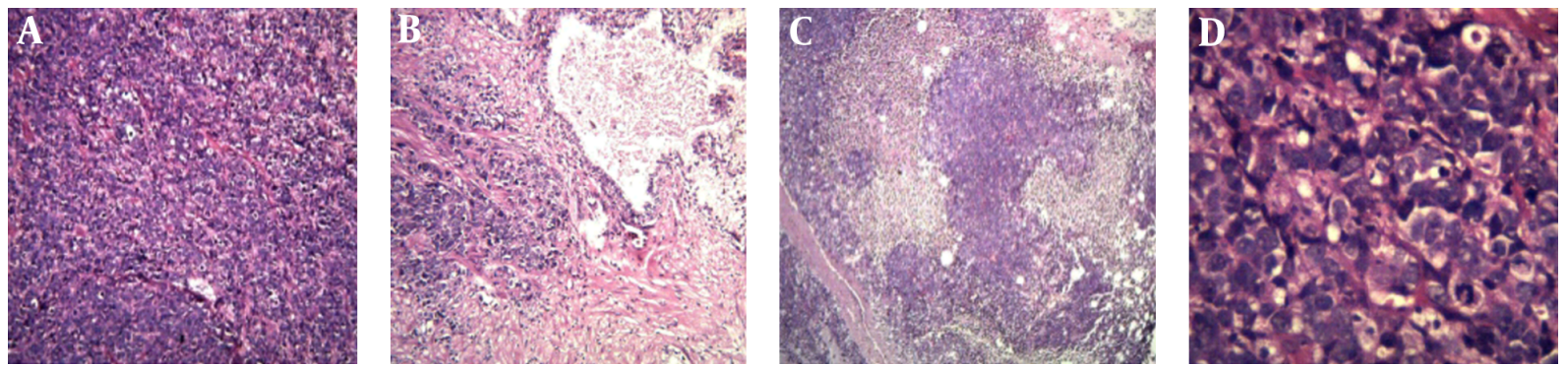

Figure 2. A, Sheet of Neoplastic Cells; B, Infiltrating Prostate Parenchyma; C, with Geographic Necrosis; D, The Neoplastic Cells had Prominent Nucleoli.

present in young males and might have a genetic base (7, 11). Animal model studies showed that prostate neuroendocrine cells could show a malignant transformation, as well (12). Some de novo LCNEC cases express androgen receptor (AR) and might be androgen dependent but other cases are AR negative (2). The main and exact mechanism and underlying causes of LCNEC are unknown.

In our case, CD56 was positive and Evans showed that this immunohistochemical marker was positive in all LCNEC patients (6). It was reported in previous studies that LCNEC cells expressed CD56, chromogranin, and synaptophysin (10). LCNEC can be diagnosed if one of the markers become positive. The first report of LCNEC Immunohistochemistry findings was published by Wynn et al. in 2000 (12). And this marker should be evaluated in suspected cases.

Travis et al. described LCNEC by specific immunohistochemistry (IHC) and electron microscopy (EM) features. He studied 5 cases of LCNEC and showed that these patients prognosis varies between atypical carcinoid and small cell carcinoma (12). The overall survival of patients with NePCs is estimated 9 to 12 months (6). All patients in Evans's study died soon after diagnosis. It seems that increased neuroendocrine differentiation is correlated with more aggressive forms of diseases and a poor prognosis (6). Our patient was discharged from the hospital and 6 months after his discharge he was still alive.

Our patient had sever pelvic pain which might have occurred due to neural invasion, or the compression effect of large pelvic lymph nodes and huge tumor. In other case reports, large mass led to urinary retention despite of pain (13). In most cases, LCNEC has been diagnosed by delay, and tumor was not resectable. Pelvic lymph node infiltration and metastases are common in patients with LCNEC like our patient.

LCNEC responded poorly to standard NePCs chemotherapy protocols. There are some recommendations for using novel and additional treatment such as somatostatin analogues in these cases, but more cases should be evaluated to develop the exact therapeutic strategy for LCNEC (14-16).

In conclusion, considering LCNEC as a differential diagnosis in patients with prostate cancer is important. Although sometimes large cell neuroendocrine carcinoma 

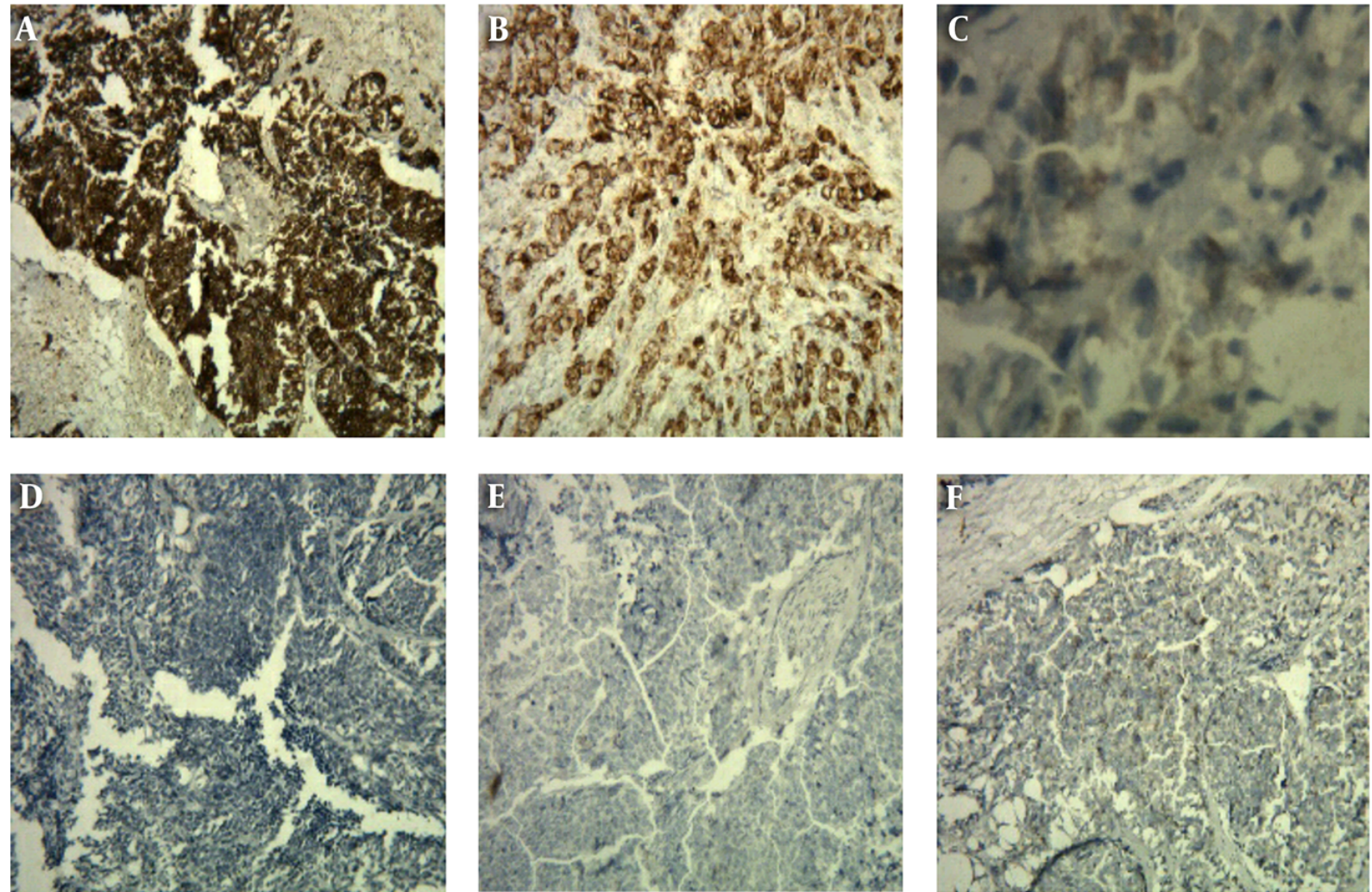

Figure 3. A, Neoplastic Cells were Positive for Synaptophysin; B, Chromogranin; C, AMACR, but Negative for D, PSA; E, GATA3; and F, CK20.

in prostate presents as metastases from other organs like lung, it is important to note the occurrence of primary large cell neuroendocrine carcinoma of prostate. On the other hand, careful histologic and IHC examination of enlarged prostate with normal PSA level must be determined in suspected cases, because it influences the prognosis and designation of the treatment strategy of the patients.

\section{Acknowledgments}

We are thankful to the research council of Mashhad University of Medical Sciences for financial support.

\section{Footnote}

Financial Disclosure: We were supported financially by the research council of Mashad University of medical sciences.

\section{References}

1. Epstein JI, Netto GJ. Biopsy interpretation of the prostate. Lippincott Williams \& Wilkins; 2008.
2. Acosta-Gonzalez G, Qin J, Wieczorek R, Melamed J, Deng FM, Zhou M, et al. De novo large cell neuroendocrine carcinoma of the prostate, case report and literature review. Am J Clin Exp Urol. 2014;2(4):337-42. [PubMed: 25606580].

3. Epstein JI, Amin MB, Beltran H, Lotan TL, Mosquera JM, Reuter VE, et al. Proposed morphologic classification of prostate cancer with neuroendocrine differentiation. Am J Surg Pathol. 2014;38(6):756-67. doi 10.1097/PAS.0000000000000208. [PubMed: 24705311].

4. Sun Y, Niu J, Huang J. Neuroendocrine differentiation in prostate cancer. Am J Transl Res. 2009;1(2):148-62. [PubMed: 19956427].

5. Humphrey PA. Histological variants of prostatic carcinoma and their significance. Histopathology. 2012;60(1):59-74. doi: 10.1111/j.13652559.2011.04039.x. [PubMed: 22212078].

6. Evans AJ, Humphrey PA, Belani J, van der Kwast TH, Srigley JR. Large cell neuroendocrine carcinoma of prostate: a clinicopathologic summary of 7 cases of a rare manifestation of advanced prostate cancer. Am J Surg Pathol. 2006;30(6):684-93. [PubMed: 16723845].

7. Azad AA, Jones EC, Chi KN. Metastatic large-cell neuroendocrine prostate carcinoma: successful treatment with androgen deprivation therapy. Clin Genitourin Cancer. 2014;12(4):e151-3. doi: 10.1016/j.clgc.2014.03.006. [PubMed: 24787970].

8. Berman-Booty LD, Knudsen KE. Models of neuroendocrine prostate cancer. Endocr Relat Cancer. 2015;22(1):R33-49. doi: 10.1530/ERC-140393. [PubMed: 25349195].

9. Shimizu K, Goto T, Maeshima A, Oyamada Y, Kato R. Prostatic metastasis of pulmonary large cell neuroendocrine carcinoma. J Cancer 2012;3:96-9. doi: 10.7150/jca.3770. [PubMed: 22359531]. 
10. Terry S, Beltran H. The many faces of neuroendocrine differentiation in prostate cancer progression. Front Oncol. 2014;4:60. doi: 10.3389/fonc.2014.00060. [PubMed: 24724054].

11. Hoof P, Tsai-Nguyen G, Paulson S, Syed A, Mora A Jr. Neuroendocrine carcinoma of the prostate gland. Proc (Bayl Univ Med Cent). 2016;29(1):68-9. [PubMed: 26722176].

12. Wynn SS, Nagabundi S, Koo J, Chin NW. Recurrent prostate carcinoma presenting as omental large cell carcinoma with neuroendocrine differentiation and resulting in bowel obstruction. Arch Pathol Lab Med. 2000;124(7):1074-6. doi: 10.1043/00039985(2000)124<1074:RPCPAO>2.0.CO;2. [PubMed: 10888786].

13. Okoye E, Choi EK, Divatia M, Miles BJ, Ayala AG, Ro JY. De novo large cell neuroendocrine carcinoma of the prostate gland with pelvic lymph node metastasis: a case report with review of literature. Int J Clin Exp
Pathol. 2014;7(12):9061-6.

14. Priemer DS, Montironi R, Wang L, Williamson SR, Lopez-Beltran A, Cheng L. Neuroendocrine Tumors of the Prostate: Emerging Insights from Molecular Data and Updates to the 2016 World Health Organization Classification. Endocr Pathol. 2016;27(2):123-35. doi: 10.1007/s12022-016-9421-z. [PubMed: 26885643].

15. Lin D, Tan AJ, De Sousa AF, Singh-Rai R. A rare case of large cell neuroendocrine carcinoma. BMJ Case Rep. 2014;2014 doi:10.1136/bcr-2014206403. [PubMed: 25331150].

16. Travis WD, Linnoila RI, Tsokos MG, Hitchcock CL, Cutler GB Jr, Nieman $\mathrm{L}$, et al. Neuroendocrine tumors of the lung with proposed criteria for large-cell neuroendocrine carcinoma. An ultrastructural, immunohistochemical, and flow cytometric study of 35 cases. Am J Surg Pathol. 1991;15(6):529-53. [PubMed: 1709558]. 